\title{
Conhecimento do Enfermeiro Obstetra no cuidado ao recém-nascido em Centros de
}

\section{Parto Normal}

\author{
Knowledge of the Nurse Obstetrics in the care of the newborn in Normal Birth Centers \\ Conocimiento de la Enfermera Obstetra em el cuidado del recién nacido em Centros Normales de \\ Nacimiento
}

Recebido: 24/05/2021 | Revisado: 31/05/2021 | Aceito: 02/06/2021 | Publicado: 17/06/2021

\author{
Naiara Lima Pereira \\ ORCID: https://orcid.org/0000-0002-5626-7690 \\ Universidade Federal do Amazonas, Brasil \\ E-mail: enf.nana@hotmail.com \\ Orácio Carvalho Ribeiro Junior \\ ORCID: https://orcid.org/0000-0003-3212-9936 \\ Universidade do Estado do Pará, Brasil \\ E-mail: oracio.junior@uepa.br \\ Kaio Vinícius Paiva Albarado \\ ORCID: https://orcid.org/0000-0002-0687-7124 \\ Universidade Federal do Oeste do Pará, Brasil \\ E-mail: kaioalbarado@gmail.com \\ Semírames Cartonilho de Souza Ramos \\ ORCID: https://orcid.org/0000-0001-8370-5994 \\ Universidade Federal da Paraíba, Brasil \\ E-mail: semiramesramosmoga@gmail.com \\ Tatiane Silva de Araújo \\ ORCID: https://orcid.org/0000-0003-1657-5565 \\ Universidade Federal do Amazonas, Brasil \\ E-mail: tatiane-araujo@live.com \\ Tayane Moura Martins \\ ORCID: https://orcid.org/0000-0003-3236-8574 \\ E-mail: tayane_m.martins1@hotmail.com \\ Universidade Federal do Pará, Brasil
}

\begin{abstract}
Resumo
Objetivo: Analisar o conhecimento dos enfermeiros quanto aos cuidados imediatos e mediatos prestados ao recémnascido nos Centros de Parto Normal (CNP) na cidade de Manaus-AM. Metodologia: Trata-se de um estudo qualitativo, descritivo, exploratório, realizado com 11 enfermeiros que atuavam no centro de Parto Normal Intrahospitalar, localizada na Zona Leste da cidade de Manaus-Am, no período de julho a setembro de 2017. Utilizou-se como instrumento de coleta de dados um roteiro de entrevista semiestruturado dividido em duas etapas. Na primeira etapa as informações diziam respeito as variáveis sexo, idade, tempo de formação e atuação. Já na $2^{\mathrm{a}}$ etapa realizou-se as perguntas abertas voltadas ao conhecimento do enfermeiro sobre os cuidados imediatos e mediatos ofertados ao recém-nascido. Realizou-se análise estatística descritiva através do RStudio com os dados da primeira etapa e análise de temática referente as perguntas abertas da segunda etapa. Resultados: Os discursos revelaram a percepção dos enfermeiros sobre os cuidados ao RN no parto e nascimento, rotinas e práticas de cuidados, e importância de capacitações voltada às ações de cuidados imediatos e mediatos ao recém-nascido sejam realizadas com maior frequência pelos enfermeiros do CPN para que situações de risco sejam minimizados nessa fase. Considerações Finais: Este estudo possibilitou analisar o conhecimento do enfermeiro obstetra relacionado os cuidados de enfermagem ao recém-nascido no Centro de Parto Normal, ocasionando uma reflexão sobre a visão desse profissional quanto à assistência ao parto e nascimento.
\end{abstract}

Palavras-chave: Recém-nascido; Enfermagem obstétrica; Salas de parto; Serviços de saúde materno-infantil.

\begin{abstract}
Objective: To analyze the nurses' knowledge regarding the immediate and immediate care provided to the newborn in the Normal Birth Centers (CNP) in the city of Manaus-AM. Methodology: This is a qualitative, descriptive, exploratory study, carried out with 11 nurses who worked at the Intra-hospital Normal Childbirth Center, located in the East Zone of the city of Manaus-Am, from July to September 2017. It used as a data collection instrument, a semistructured interview script divided into two stages. In the first stage, the information related to the variables sex, age, time since graduation and performance. In the 2 nd stage, open questions were made, aimed at the nurses' knowledge about the immediate and immediate care offered to the newborn. Descriptive statistical analysis was carried out
\end{abstract}


through RStudio with the data from the first stage and thematic analysis referring to the open questions in the second stage. Results: The speeches revealed the nurses' perception about the care of the NB during childbirth and birth, care routines and practices, and the importance of training aimed at immediate and immediate care actions for the newborn to be carried out more frequently by nurses at the ANC so that risk situations are minimized in this phase. Final Considerations: This study made it possible to analyze the knowledge of obstetric nurses related to nursing care for newborns at the Center for Normal Childbirth, causing a reflection on the view of this professional regarding assistance in childbirth and birth.

Keywords: Newborn; Obstetric nursing; Childbirth rooms; Maternal and child health services.

\section{Resumen}

Objetivo: Analizar el conocimiento de las enfermeras sobre la atención inmediata e inmediata al recién nacido en los Centros de Parto Normal (CNP) de la ciudad de Manaus-AM. Metodología: Se trata de un estudio cualitativo, descriptivo, exploratorio, realizado con 11 enfermeras que laboraron en el Centro Intrahospitalario de Parto Normal, ubicado en la Zona Este de la ciudad de Manaus-Am, de julio a septiembre de 2017. Se utilizó como un instrumento de recolección de datos, un guión de entrevista semiestructurado dividido en dos etapas. En la primera etapa, la información relacionada con las variables sexo, edad, tiempo desde egreso y desempeño. En la $2^{\mathrm{a}}$ etapa se realizaron preguntas abiertas, orientadas al conocimiento de las enfermeras sobre la atención inmediata e inmediata que se brinda al recién nacido. El análisis estadístico descriptivo se realizó a través de RStudio con los datos de la primera etapa y el análisis temático referido a las preguntas abiertas en la segunda etapa. Resultados: Los discursos revelaron la percepción de las enfermeras sobre el cuidado del RN durante el parto y el parto, las rutinas y prácticas de atención, y la importancia de la formación orientada a las acciones de atención inmediata e inmediata al recién nacido a ser realizadas con mayor frecuencia por las enfermeras del CPN para que las situaciones de riesgo se minimicen en esta fase. Consideraciones finales: Este estudio permitió analizar los conocimientos de las enfermeras obstétricas relacionadas con el cuidado de enfermería del recién nacido en el Centro de Parto Normal, provocando una reflexión sobre la visión de esta profesional sobre la asistencia en el parto y el parto.

Palabras clave: Recién nacido; Enfermería obstétrica; Salas de partos; Servicios de salud materno-infantil.

\section{Introdução}

O período neonatal é caracterizado por uma fase de vulnerabilidade a riscos biológicos, ambientais, sociais e culturais, pelos quais passam os recém-nascidos. (Pinheiro et al., 2016). Nesse sentido infere-se a necessidade de que cuidados imediatos e mediatos sejam capazes de reduzir índices de mortalidade nessa fase. Segundo Pereira et al. (2017), o período de maior risco para mortalidade infantil é o neonatal precoce, sendo de difícil decréscimo devido as estratégias a serem implementadas na qualificação durante o pré-natal e, até mesmo, durante o parto.

Os níveis de mortalidade neonatal no Brasil vêm se mantendo em índices elevados, com taxa de 11,2 óbitos por mil nascidos vivos em 2010 (Lansky et al., 2014). De acordo com dados do MS, no período de 2000 a 2011 ocorreu uma média de 13,5 óbitos por mil nascidos vivos na região norte. Porém o estado do Amazonas apresentou média superior comparado ao da região norte com taxa de 15,9 óbitos (Brasil, 2012a).

Como estratégia de reafirmação de todas as políticas públicas de saúde voltadas à mulher e a criança, a Rede Cegonha foi lançada em 2011, com objetivo de propor mudanças significativas quanto ao modelo hegemônico de assistência ao parto e nascimento no Brasil. O programa busca devolver à mulher a figura de protagonista do processo de parturição, fazendo deste momento uma experiência íntima e pessoal, dentro de uma ambiência adequada para a boa evolução do nascimento (Brasil, 2014). No intuito de oferecer uma assistência adequada e de maior qualidade ao neonato, em 2014 o Ministério da Saúde disponibilizou o manual de atenção ao recém-nascido e a Portaria 371/2014 que institui diretrizes para a organização da atenção integral e humanizada ao RN no Sistema Único de Saúde (SUS) (Brasil, 2014a, 2014b).

A assistência adequada no momento do nascimento e nas primeiras horas é fundamental para a redução de riscos à saúde do recém-nascido. Os principais objetivos dos cuidados imediatos são proporcionar ao RN as condições ideais para adaptação à vida extrauterina (Farias, Souza \& Morais, 2020). 
Para Peppe (2017), o profissional de enfermagem é membro ativo na promoção de cuidados humanísticos, auxiliando no momento do parto e instituindo tecnologias não farmacológicas de cuidado e conforto à parturiente e ao recém-nascido, contribuindo com a oferta de cuidados adequados ao RN.

Assim, o interesse por realizar esse estudo nasceu da vivência da pesquisadora durante as práticas assistenciais realizadas no curso de Residência em Enfermagem Obstétrica da Universidade Federal do Amazonas, sendo este um cenário ideal para contato e convívio com enfermeiros obstetras responsáveis pelas demandas de cuidados aos recém-nascidos.

Vale considerar que a pesquisa vai ao encontro das diretrizes indicadas pela rede cegonha, que busca a redução da mortalidade materna e neonatal, assegurando o direito ao nascimento seguro por meio de uma assistência de qualidade ao Recém-nascido. $\mathrm{O}$ estudo tem o intuito de contribuir para promover melhorias e reflexões sobre a assistência ao recém-nascido em sala de parto, bem como o pensamento crítico do profissional acerca do tema, elucidando a necessidade de reverem ou não suas práticas.

Frente ao exposto, foi estabelecido como objeto de estudo analisar o conhecimento dos enfermeiros quanto aos cuidados imediatos e mediatos prestados ao recém-nascido nos Centros de Parto normal (CNP) na cidade de Manaus-AM, justificandose pela importância de refletir sobre o conhecimento do enfermeiro obstetra frente às demandas exigidas pelo Ministério da Saúde quanto à assistência ao RN.

\section{Metodologia}

Trata-se de uma pesquisa de abordagem qualitativa, de natureza descritiva, que buscou analisar o discurso de enfermeiros obstetras sobre os cuidados imediatos e mediatos ao recém-nascido nos Centros de Parto Normal. A abordagem qualitativa permite uma análise mais aprofundada do objeto de estudo, preocupando-se com a realidade, com significados, motivos, crenças, além de valores e atitude. Este tipo de abordagem é ideal para investigação de grupos, de histórias sociais sob a ótica dos próprios sujeitos (Minayo, 2010). Escolheu-se pela pesquisa descritiva se deu pelo fato de as pesquisas descritivas terem como objetivo primordial a descrição das características de determinada população ou fenômeno (Gil, 2008).

A pesquisa foi realizada em uma maternidade pública vinculada à rede cegonha, que possui Centro de Parto Normal Intra-hospitalar, localizada na Zona Leste da cidade de Manaus. Inaugurada no dia 10 de maio de 2004, sendo também credenciada como Hospital Amigo da Criança, título concedido pela Organização Mundial de Saúde (OMS) e Fundo das Nações Unidas para a Infância (Unicef).

Participaram da pesquisa 11 enfermeiros que atuavam no centro de Parto Normal no período da coleta de dados nos meses de julho a setembro de 2017. Os enfermeiros foram esclarecimentos sobre os objetivos e métodos da pesquisa e após a leitura do Termo de Consentimento Livre e Esclarecido - TCLE assinaram confirmando a anuência em participar da pesquisa. Foram incluídos nesta pesquisa os enfermeiros que possuíssem especialização em enfermagem obstétrica e que atuassem na assistência ao parto e nascimento nos Centros de Parto Normal. Os profissionais que no período da coleta estivessem de licença médica, licença maternidade ou em gozo de férias, não puderam participar do estudo.

Então, utilizou-se como instrumento de coleta de dados um roteiro de entrevista semiestruturada dividida em duas etapas: Na $1^{\mathrm{a}}$ etapa constituiu-se a caracterização do enfermeiro, referente a sexo, idade, tempo de formação e atuação. Já na $2^{\mathrm{a}}$ etapa realizou-se as perguntas abertas voltadas ao conhecimento do enfermeiro sobre os cuidados imediatos e mediatos ofertados ao RN no CPN tomando como base as recomendações do Ministério da Saúde quanto aos cuidados imediatos e mediatos ao recém-nascido. A escolha pela entrevista se deu pelo fato desta fornecer, como fonte de informação, dados que se referem diretamente ao indivíduo entrevistado, que tratam de sua reflexão sobre a realidade e constituem uma representação da realidade (Minayo, 2010). As entrevistas foram gravadas a fim de garantir a autenticidade das falas e duravam em média de 15 
a $20 \mathrm{~min}$, sendo transcritas na íntegra, para posterior análise. Os dados referentes a primeira etapa, foram organizados e analisados através do software RStudio destacando-se as médias $(M)$ e o desvio padrão $(D P)$ dessas variáveis.

No entanto as respostas aos enunciados da segunda etapa, foram organizados e analisados através das falas por meio da técnica de análise temática. Essa análise possui função de apreender as falas, explicitas ou implícitas nos discursos dos sujeitos da pesquisa (Minayo, 2010).

Operacionalmente, segundo Minayo (2010), uma pesquisa passa por três fases: Pré-análise, fase de exploração do material e tratamento dos resultados. A primeira fase, consistiu-se na organização do material coletado por meio da exploração do material, a partir da leitura flutuante, que requer do pesquisador um contato direto e intenso com o material coletado. A segunda etapa, refere-se à exploração do material, em que os dados brutos são codificados para se alcançar o núcleo de compreensão do texto. A codificação envolve procedimentos de recorte, contagem, classificação, desconto ou enumeração em função de regras previamente formuladas (Minayo, 2010). A terceira e última etapa se refere ao tratamento dos resultados obtidos e posterior interpretação, em que o pesquisador propõe inferências e realiza interpretações, relacionando-as ao quadro teórico desenhado inicialmente ou abre outras pistas em torno de novas dimensões teóricas e interpretativas, sugeridas pela leitura do material (Minayo, 2010).

A pesquisa atendeu a Resolução 466/2012, sendo aprovada pelo comitê de ética e pesquisa da Escola de Enfermagem de Manaus da Universidade Federal do Amazonas sob o CAEE $n^{\circ}$ 2.141.794. Para a preservação do anonimato, os participantes foram identificados por meio de letras e números que os identificavam como enfermeiros E1, E2..., sendo a letra "E" referente a palavra enfermeiro e a numeração referente a ordem de realização das entrevistas.

\section{Resultados e Discussão}

Os participantes foram 11 enfermeiros, sendo 9 (82\%) do sexo feminino e 2 (18\%) do sexo masculino. Segundo o Conselho Federal de Enfermagem, no ano de 2011, a maioria dos profissionais de enfermagem eram mulheres, correspondendo a $88,02 \%$ desses profissionais no Brasil. A enfermagem sempre possuiu uma estreita ligação com o feminino, visto que historicamente as mulheres sempre desempenharam o papel de cuidadoras. Para Passos (2012) a mulher é vista pela sociedade como possuidora de condições naturais para zelar, promover e ajudar o indivíduo a se desenvolver harmoniosamente, essas supostas tendências femininas fazem com que sejam atribuídos a elas o papel do cuidado de enfermos. Essa tendência continua sendo vista atualmente, em que o número de profissionais de enfermagem do sexo feminino continua sendo superior nessa área.

Em relação à idade, os resultados evidenciaram que a maioria dos profissionais participantes da pesquisa são de meiaidade, entre 31 a 50 anos, correspondendo a $84 \%$ da amostra. Os profissionais mais jovens com faixa etária, entre 20 e 30 anos, correspondem a $11 \%$ desses profissionais, enquanto apenas $5 \%$ representavam a faixa etária com idade superior a 50 anos de idade $(M=36,8 ; D P=8,9)$.

Analisando os dados sobre a faixa etária, percebe-se uma predominância de enfermeiros com idade inferior a 40 anos (55\%). Resultados semelhantes foram encontrados no estudo de Andrade et al. (2017) realizado com 12 profissionais da enfermagem, e representavam uma média de 36 anos de idade. Provavelmente a maioria desses profissionais seja composta por um público jovem.

Quanto ao tempo de formação, $64 \%$ dos enfermeiros possuíam mais de cinco anos de formação $(M=7,4 ; D P=6,2)$. Sobre o tempo de atuação desses profissionais, $55 \%$ possuíam mais de três anos de atuação, sugerindo tempo suficiente para o desenvolvimento de experiência profissional na área de enfermagem obstétrica $(M=6,6 ; D P=6,2)$. Observa-se que esses últimos escores são próximos devido a proximidade do tempo de formação com a experiência profissional, supondo que uma boa parte dos entrevistados terminaram a graduação e conseguiram se inserir no mercado de trabalho de imediato. 
Corroborando a isso, um estudo realizado por Luz et al. (2020), que teve por finalidade avaliar a qualidade de vida da equipe de profissionais de saúde atuantes no setor do Centro Obstétrico, a maioria desses profissionais nesse setor declararam atuar entre de 1 a 3 anos, totalizando 38,5\% dos sujeitos. Verifica-se uma aproximação do período de formação e o tempo de atuação, demonstrando uma enfermagem obstétrica mais jovem atualmente. Cabe ressaltar que a experiência de outros profissionais com mais anos de carreira torna-se essencial para o bom andamento nos serviços de enfermagem. Esses jovens atores da saúde, tem muito para aprender como os mais experientes.

Após a realização da análise de conteúdo de Minayo, os temas emergentes foram agrupados em quatro categorias: I - A Assistência ao parto e nascimento é uma caixinha de surpresas. II- Promoção ao contato pele a pele na primeira hora de vida. III - Até parar de pulsar: a importância do clampeamento tardio do cordão e IV - Rotina no cuidado com o recém-nascido.

\subsection{A Assistência ao parto e nascimento é uma caixinha de surpresas}

Os enfermeiros obstetras compreendem que cada parto tem suas peculiaridades e entendem a importância de um preparo na área de atuação para maior resolutividade nas emergências no parto e cuidados ao recém-nascido. Observa-se que em sua maioria os recém-nascidos de partos de risco habitual apresentam boa vitalidade, não eximindo a necessidade de que os profissionais que atuam no atendimento ao parto e nascimento tenham conhecimento e habilidade profissional para atender esse recém-nascido. Para os participantes deste estudo o enfermeiro necessita estar preparado para atender as possíveis intercorrências no parto e nascimento, como expresso nas falas abaixo:

"Porque o parto pode ser mais fisiológico possivel, mas ele é uma caixinha de surpresas e alguma coisa sempre pode dar errado e se acontecer ele tem que tá preparado pra isso." (E4)

"Ele tem que ter noções e saber mesmo sobre reanimação neonatal, porque o parto é uma caixinha de surpresas, o bebê tá ali bem e quando nasce a gente pode ter uma surpresa (...) então a gente tem saber reanimar o bebê dentro dos critérios que o ministério da saúde preconiza" (E3)

"Ele precisa ter conhecimentos também de manobras de reanimação, e é por isso que é importante fazer os cursos de reanimação da sociedade Brasileira de Pediatria, saber reanimar tanto o recém-nascido a termo como o pré-termo, saber os parâmetros de boa vitalidade e de má-vitalidade e as manobras iniciais" (E11)"

Tanus e Carneiro (2018), afirmam que os profissionais de enfermagem devem estar com os conhecimentos alinhados no que se refere aos cuidados imediatos no recém-nascido. Dessa forma, verifica-se a importância de educação continuada e treinamentos para a equipe, permitindo uma maior capacidade para executar ações que proporcionem eficiência e eficácia no atendimento.

Os participantes do estudo percebem ainda que existem diferenças nos cuidados imediatos na sala de parto aos recémnascidos com boa vitalidade e do recém-nascido que não possui frequência respiratória e cardíaca favoráveis no primeiro momento de vida, sabendo que este necessita de cuidados distintos ao nascer.

"Primeiramente deve conhecer tudo que é normal, a parte fisiológica de um RN, para depois determinar, para depois você avaliar se você tá entrando em alguma alteração, alguma distorcia, alguma anormalidade no RN. (...) também tá preparado quando se apresenta isso. Você que atende o parto, quais as medidas que você vai tomar? O que você vai fazer passo a passo? Sobre o que é reanimação, o que é o atendimento inicial ao RN?" (E2). 
"Saber distinguir né? O que é um RN fisiológico e o patológico, por que são cuidados totalmente diferentes, a gente sabe que quando é um RN fisiológico a gente não precisa exceder os cuidados (E4)”

"Saber avaliar a vitalidade do recém-nascido ao nascimento, frequência cardíaca, esforço respiratório e tônus muscular, principalmente frequência cardíaca e padrão respiratório que são os dois critérios da sociedade para saber se o recém-nascido precisa ou não de manobras de reanimação, então o primeiro passo é ele saber essas informações sobre a vitalidade do recém-nascido" (E11)

Um dos objetivos da rede-cegonha do Ministério da Saúde é a capacitação de profissionais para atuar em emergências, dentre elas a reanimação neonatal no parto-nascimento, para garantir que o recém-nascido respire no primeiro minuto de vida, também chamado de Minuto de Ouro. No Brasil estima-se que, a cada ano, 300 mil crianças irão precisar de suportes ao nascer para iniciar e manter a respiração (Brasil, 2012).

Almeida e Guinsburg (2013) apontam que, quando se espera recém-nascidos hígidos, sem hipóxia ou asfixia é necessário que os profissionais tenham habilidade para atuar em situações que seja necessário a reanimação neonatal. Essa preocupação se deve a morte de mais de 13 recém-nascidos, entre os anos de 2005 e 2009, devido a condições associadas à asfixia perinatal.

Nesse sentido tais dados trazem a reflexão acerca da visão do profissional relacionada à importância de um olhar atento as especificidades dessa fase e está preparado para situações adversas na assistência ao parto e nascimento, pois um atendimento inicial adequado no minuto de ouro, garante que os recém-nascidos recebam cuidados emergenciais livre de sequelas ou óbito por falta de uma assistência segura.

\subsection{Promoção do contato pele a pele na primeira hora de vida}

No contato pele a pele, o recém-nascido com boa vitalidade deve ser colocado imediatamente sobre o tórax da mãe, devendo os profissionais proporcionar o aleitamento materno imediato (Lins, 2017). Isso é evidenciado no discurso dos enfermeiros deste estudo, demonstrando a compreensão acerca da importância do contato pele a pele no primeiro momento de vida do recém-nascido.

“Em contato pele a pele e lá mesmo ela já se preocupa com o primeiro momento que é de aquecer, de enxugar primeiramente ele, principalmente a cabecinha dele, enxugar logo e aquecer em seguida e fazer aquele contato (...). o $R N$ tá ativo e reativo, tá respondendo legal, a gente deixa no contato pele a pele” (E1)

"Eu acho que o importante quando nasce é colocar em contato pele a pele (...) o contato pele a pele pra que se tenha mais vínculo entre a mãe e o bebê, e ela fique consciente de que o seu filhinho tá bem e fique satisfeita. Com isso vai até melhorar fisiologicamente e emocionalmente o pós-parto dela” (E10)

“Eu vou fazer primeiramente o contato pele a pele imediato, então tem aquele contato, aquele vinculo mãe e bebê, a gente sabe que após o nascimento, a visão dele nos próximos minutos é limitada, então a distância que ele consegue ver é em torno de 30 a 35 centímetros, que é a distância do peito pra face da mãe, então é por isso e não é à toa que que fazemos o contato pele a pele e já coloca o peito materno, é porque o primeiro contato visual dele tem que ser com a mãe até por conta da limitação , que tudo isso é algo fisiológico e natural." (E11) 
O contato pele a pele ajuda a estabilizar os níveis de sangue, nos valores da frequência cardíaca e incursão respiratória do neonato, além da redução do choro e do estresse do recém-nascido para que se obtenha menor perda de energia além de manter o bebê aquecido pela transmissão de calor (Almeida \& Martins Filho, 2004).

Corroborando a isso, Campos et al. (2020), ao realizar uma pesquisa com 586 mulheres em um centro de internação obstétrica no Rio Grande do Sul, verificou-se que $60,1 \%$ receberam o estímulo para a realização do contato pele a pele e $44 \%$ foram estimuladas a realizar a amamentação materna imediata ao parto. Isso reforça a importância da realização dessa prática incitado pelo próprio obstetra.

Os profissionais compreendem a importância desse momento, fazendo os cuidados mediatos após a primeira hora do nascimento, reconhecendo a relevância do vínculo estabelecido entre mãe e recém-nascido, como relatado nos discursos abaixo:

"Se ele tá bem eu deixo por uma hora, depois de uma hora a gente dá os cuidados básicos, que são antropométricos, a gente vai medir e pesar, vai fazer o clampeamento do coto, fazer o curativo e colocar ele no peito" (E3)"

"A gente pode ficar até uma hora com ele ali. Então pode ser depois, não tem isso de ser agora. Nasceu já tem que cortar e fazer esses cuidados? Não!" (E9)

"Se a mãe já tiver com os exames de rotina liberados a gente já pode colocar esse bebê pra sugar, pra estimular a sucção naquele momento, pra formar aquele vínculo, né? Aquele vínculo entre a mãe o bebê que é primordial também, aquele reconhecimento do campo que ele vai pelo instinto reconhecer o campo, o seio materno, o bico do seio, o mamilo, então ele já vai identificar a sucção" (E4)

Ainda de acordo com estudo realizado por Campos et al. (2020) os benefícios do contato precoce pele-a-pele para a mãe e recém-nascido pode permitir um momento de tranquilidade e segurança, sendo vantajoso para ambos. Dessa forma, esse momento de interação pode proporcionar os efeitos fisiológicos benéficos a puérpera.

O contato pele a pele é tido como um passo fundamental para estimular o aleitamento materno. Vale considerar que a maternidade pesquisada faz parte da Iniciativa Hospital Amigo da Criança (IHAC) que apoia o aleitamento materno na primeira hora de vida. De acordo com essa iniciativa, proporcionar a amamentação imediatamente ao nascer será um passo para o sucesso na saúde do RN (Boccolini,2011).

A discrepância entre as evidências cientificas atuais sobre a importância do contato pele a pele e as práticas assistenciais rotineiras nas maternidades, foram apontadas no estudo de Sampaio, Bousquat e Barros (2016) onde foi verificado que somente $9,3 \%$ das mulheres de uma maternidade pública no Nordeste tiveram a oportunidade de manter o contato pele a pele com seus bebês na primeira hora de vida. Nesse caso, percebe-se que alguns profissionais podem não estar estimulando essas práticas com as mães no pós-parto imediato.

A promoção do contato pele a pele pelos profissionais de saúde e estímulo à amamentação reflete o respeito pelo binômio mãe-filho, compreendendo sua importância e minimizando intervenções desnecessárias ao RN. Isso pode fazer com que a recepção seja mais segura e tranquila. De certa forma, o Brasil (2012b) preconiza que o contato pele a pele deve ser realizado após o nascimento, sendo esse também um momento propicio para promover a amamentação.

\subsection{Até parar de pulsar: a importância do clampeamento tardio do cordão}

A anemia infantil, tem como uma das principais causas a deficiência de ferro, que aumenta a mortalidade infantil, causando problemas de desenvolvimento cognitivo, motor e comportamental (Zavaleta \& Robilliard, 2017). Nesse sentido os enfermeiros participantes do estudo compreendem tal importância, como evidencia-se nos discursos abaixo: 
"Outro procedimento muito importante que tá sendo adotado é o corte do cordão umbilical tardio, né? A gente só corta mesmo quando a gente vê que já parou de pulsar, entende? a gente se policia muito nisso" (E1)

"A gente espera ali terminar de pulsar o cordão, cessar a pulsação do cordão e faz o clampeamento, a gente faz o clampeamento, o corte e faz a coleta de sangue do cordão, isso enquanto ele tá no contato pele a pele" (E4)

"Clampeamento oportuno que é estabelecido hoje pelo ministério que é 3 minutos... até 3 minutos tem que esperar para fazer o clampeamento se ele nasce com boa vitalidade né? Visto que esse suprimento que ele recebe nesses 3 minutos vai ser benéfico pra ele lá na frente pra ele não ter anemia na primeira infância, tá certo?!” (E11)

Segundo Oliveira et al. (2014) o clampeamento tardio é aquele realizado após três minutos do nascimento ou assim que cessarem as pulsações do cordão. Em uma revisão integrativa da literatura, realizada por Navarro (2016), identificou os benefícios descritos nos estudos, como maiores valores no nível de ferritina, menor prevalência de hematócrito baixo no recém-nascido, sendo assim, uma estratégia fundamental de combate à anemia ferropriva, apresentando baixo custo e praticidade. Com isso, tal prática não gera complicações na adaptação cardiovascular e respiratória nos recém-nascidos e nem alterações nas puérperas.

O consenso entre os enfermeiros na prioridade do clampeamento tardio do cordão foi apontado no estudo de Muller (2012), demonstrando uma sensibilização por parte dos enfermeiros obstetras para que essa recomendação seja concretizada na assistência ao parto e nascimento.

Porém, o tempo exato indicado para essa prática ainda pode gerar controvérsias entre esses profissionais. De acordo com. Sarli (2018) os bebês que apresentaram icterícia neonatal tiveram um tempo médio de clampeamento do cordão umbilical após o nascimento de 56,5 segundos, contra 50,4 segundos dos RN sem icterícia. Além disso, o mesmo autor afirma houve correlações positivas entre o período para o clampeamento do cordão e a duração da fototerapia. Dessa forma, ao se passar 1 segundo para a realização do clampeamento, aumenta em 9 minutos a duração de fototerapia.

\subsection{Rotina nos cuidados com o recém-nascido.}

Segundo Tanus e Carneiro (2018) as rotinas e normas hospitalares influenciam o comportamento dos enfermeiros, sendo que no ambiente hospitalar o recém-nascido está exposto a diversas intervenções e grande parte delas são realizadas pelo profissional de enfermagem. Os participantes descrevem os cuidados de rotina ao recém-nascido sem intercorrências como relatadas nas falas abaixo:

"Faz o exame físico né? de fontanelas, do dorso, se ele tá respondendo aos reflexos, verifica logo a respiração, se tá regular, olha a parte genital, daí em seguida a gente faz o crede né? vitamina $K$, faz a mensuração principalmente da parte cefálica, dos demais ... do tórax, abdômen, daí a gente em seguida a gente põe para pesar o RN, aí daí sim, a gente enxuga novamente, deixa tudo limpinho, arruma o bebê e devolve pra na mãe” (E1)

"A gente pega o bebê, seca, faz os primeiros cuidados imediatos, faz a mensuração, crede, avalia tônus desse bebê, depois a gente leva contato pra mãe, para as primeiras horas de amamentação." (E7)

"A verificação do perímetro cefálico, torácico e abdominal, peso, altura, cuida do coto umbilical, vê se tem duas artérias e uma veia, faz a vitamina $K$, faz curativo no coto umbilical, aquece o bebê que é muito importante ficar aquecido orienta a mãe sobre a importância de ficar aquecido." (E8) 
Observa-se que existe rotina quanto aos cuidados com o recém-nascido, sendo consenso entre os profissionais a importância do exame físico, secar, aquecer, realizar as medidas antropométricas, administração do nitrato de prata, administração de kanakion, cuidado com o coto umbilical e o estímulo à amamentação. Esses mesmos cuidados de rotina ao RN foram relatados no estudo do Müller (2012).

Muller (2012) também aponta os procedimentos que devem ser realizados na assistência ao recém-nascido, como: 1) a laqueadura do coto umbilical com higienização com álcool a 70\% ou clorexidina alcoólica a $0,5 \%$; ) a prevenção da oftalmia gonocócica pelo método de crede afastando a pálpebra e instalando o nitrato de prata;3) a antropometria; e 4) a administração da vitamina K para prevenção de hemorragia. A identificação é feita por meio de pulseiras colocadas na mãe e no RN contendo o nome da mãe, registro hospitalar, data e hora do nascimento e o sexo do recém-nascido.

Compreende-se que os profissionais seguem as rotinas conforme as preconizações para conduzir os cuidados ao recémnascido, o que demonstra a compreensão e importância das práticas realizadas para uma recepção e adaptação ao meio extrauterino de forma segura.

\section{Conclusão}

O estudo revelou, um panorama quanto à caracterização do perfil dos enfermeiros obstetras que atuam na assistência ao recém-nascido nos centros de parto normal e seus conhecimentos relacionados aos cuidados direcionados ao recém-nascido. As análises das falas dos participantes do estudo sobre a assistência prestada ao RN apontam conformidade com os cuidados preconizados nas políticas de atenção ao recém-nascido.

Observa-se a insegurança e a busca por aprimoramento das questões relacionadas à reanimação neonatal na sala de parto, cabendo à instituição cenário deste estudo, a oferta por cursos de aperfeiçoamento para garantir maior segurança na prática profissional, com o estabelecimento de treinamentos e protocolos de assistência capazes de suprir as necessidades de aprendizado dos profissionais e consequentemente a promoção de uma assistência qualificada e segura ao recém-nascido.

Os enfermeiros participantes do estudo, reconhecem a importância de cuidados humanizados, com respeito à hora dourada, o contato pele a pele e clampeamento oportuno do cordão. Sendo um grande passo haja vista a criação dos centros de parto normal estar diretamente vinculadas ao aumento e incentivo às práticas que beneficiarão a mãe, o recém-nascido e a família.

Ressalta-se o consenso entre os enfermeiros participantes do estudo quanto os cuidados humanizados, contato pele a pele e clampeamento oportuno do cordão. Porém aponta-se a necessidade de que mais estudos de cunho experimental sobre o contato pele a pele e clampeamento do cordão sejam realizados, assim como pesquisas voltadas para a prática assistencial dos enfermeiros ao RN com um número de sujeitos maior, com a finalidade de minimizar as divergências entre o discurso e as práticas assistenciais.

Portanto, este estudo possibilitou analisar o conhecimento do enfermeiro obstetra no que tange os cuidados de enfermagem ao recém-nascido no Centro de Parto Normal, ocasionando uma reflexão sobre a visão do enfermeiro obstetra quanto à assistência ao parto e nascimento. Com isso, discute-se a importância desses profissionais como mediadores no processo de implementação do parto e de cuidados humanizados ao RN.

\section{Referências}

Andrade, L. O. de., Felix, E. Da S. P., Souza, F. S., Gomes, L. O. S., \& Boery, R. N. S. De O. (2017). Práticas dos profissionais de enfermagem diante do parto humanizado. Revista de Enfermagem da UFPE on line. 11(6), 2576-2585. https://: 10.5205/reuol.9799-86079-1-RV.1106sup201712.

Almeida, E. A. De., \& Martins Filho, J. (2004). O contato precoce mãe-filho e sua contribuição para o sucesso do aleitamento materno. Revista de Ciências Médicas. 13(4), 381-388. http://seer.sis.puc-campinas.edu.br/seer/index.php/cienciasmedicas/article/viewFile/1214/1189. 
Almeida, M.F.B De., \& Guinsburg, R. (2013). Reanimação Neonatal em Sala de Parto: Documento Científico do Programa de Reanimação Neonatal da Sociedade Brasileira de Pediatria. Sociedade Brasileira de Pediatria. www.sbp.com.br.

Boccolini, C. S., Carvalho, M. L. De., Oliveira, M. I. C. De., \& Vasconcellos, A. G. G. .(2011).Fatores associados à amamentação na primeira hora de vida. Revista de Saúde Pública. 45 (1), 69-78. https://doi.org/10.1590/S0034-89102010005000051.

Brasil. Ministério da Saúde. Datasus. (2012a). http://tabnet.datasus.gov.br/cgi/idb2012/c0104b.htm.

Brasil. Ministério da Saúde. (2012b). Secretaria de Atenção à Saúde. Atenção à saúde do recém-nascido: guia para os profissionais de saúde. https://bvsms.saude.gov.br/bvs/publicacoes/atencao_saude_recem_nascido_profissionais_v1.pdf

Brasil, Ministério da Saúde Portaria N ${ }^{o}$ 371/2014. (2014b). http://bvsms.saude.gov.br/bvs/saudelegis/sas/2014/prt0371_07_05_2014.html.

Brasil. Ministério da Saúde. (2014a). Atenção à saúde do recém-nascido. Guia para os profissionais da saúde. http://bvsms.saude.gov.br/bvs/publicacoes/atencao_saude_recem_nascido_v1.pdf.

Campos, P. M., Gouveia, H. G., Strada, J. K. R., \& Moraes, B. A. (2020). Contato pele a pele e aleitamento materno de recém-nascidos em um hospital universitário. Revista Gaúcha de Enfermagem. 41(esp), 1-10. https://doi.org/10.1590/1983- 1447.2020.20190154.

Farias, R. V., Souza, Z. C. S. Do N., \& Morais, A. C. (2020). Prática de cuidados imediatos ao recém-nascido: uma revisão integrativa de literatura. Revista Eletrônica Acervo Saúde. 56. 1-10. https://doi.org/10.25248/reas.e3983.2020.

Gil, A. C. (2008). Métodos e Técnicas de Pesquisa Social. Atlas.

Lansky, S., Friche, A. A. De L., Silva, A. A. M. Da., Campos, D., Bittencourt, S. D. De A., Carvalho, M. L. De., Frias, P. G. De., Cavalcante, R. S., \& Cunha, A. J. L. A. Da. (2014). Pesquisa Nascer no Brasil: perfil da mortalidade neonatal e avaliação da assistência à gestante e ao recém-nascido. Cadernos de Saúde Pública .30 (1), 192-207. https://doi.org/10.1590/0102-311X00133213.

Lins, J. W. C J. Da S. (2017). Implantação do protocolo de boas práticas na assistência ao recém-nascido na sala de parto. [Trabalho de Conclusão de Curso, Universidade Federal de Minas Gerais].

Luz, A. De M., Messias, A. L. B., Ribeiro, E. S., Lima, L. F., Fernandes, C. O., \& Kawakame, P. M. G. (2020). Qualidade de vida dos profissionais de saúde atuantes no centro obstétrico. Perspectivas Experimentais e Clínicas, Inovações Biomédicas e Educação em Saúde. 6(2),1-9. https://trilhasdahistoria.ufms.br/index.php/pecibes/article/view/12244.

Matos, T. A., Souza, M. S. De., Santos, E. K. A. Dos., Velho, M. B., Seibert, E. R. C., \& Martins, N. M. (2010). Contato precoce pele a pele entre mãe e filho: significado para mães e contribuições para a enfermagem. Revista Brasileira de Enfermagem. 63 (6), 998-1004. https://doi.org/10.1590/S003471672010000600020

Minayo, M. C. De S. (2010). O desafio do conhecimento: pesquisa qualitativa em saúde. Hucitec.

Müller, E. B. (2012). Cuidados ao recém-nascido no centro obstétrico: uma proposta de enfermeiras com base nas boas práticas. [Dissertação de Mestrado Profissional em Gestão do Cuidado em Enfermagem da Universidade Federal de Santa Catarina].

Navarro, C.R.P (2016). O que diz a literatura sobre o clampeamento tardio do cordão umbilical no recém-nascido a termo: argumentos para implementação de boas práticas. [Trabalho de Conclusão de Curso da Escola de Enfermagem da Universidade Federal do Rio Grande do Sul].

Oliveira, F. De C. C., Assis, K. F., Martins, M. C., Prado, M. R. M. C. Do., Ribeiro, A. Q., Sant'Ana, L. F. Da R., Priore, S. E., \& Franceschini, S. Do C. C. (2014). Tempo de clampeamento e fatores associados à reserva de ferro de neonatos a termo. Revista de Saúde Pública. 48(1), 10-18. https:// 10.1590/S00348910.2014048004928.

Passos, E. (2012). De Anjos a Mulheres: ideologias e valores na formação de enfermeiras. EDUFBA.

Pereira, M. U. L., Lamy Filho, F., Anunciação, P. S. Da., Lamy, Z. C., Gonçalves, L. L. M. G., \& Madeira, H. G. R. (2017). Óbitos neonatais no município de São Luís: causas básicas e fatores associados ao óbito neonatal precoce neonatal. Revista de Pesquisa em Saúde.18 (1), 18-23. http://www.periodicoseletronicos.ufma.br/index.php/revistahuufma/article/view/7874.

Peppe, M.V (2017). A assistência puerperal prestada pelas enfermeiras obstetras elou obstetrizes que realizam o parto domiciliar planejado no estado de São Paulo. [Dissertação de Mestrado, Universidade de São Paulo].

Pinheiro, J. M. F., Tinoco, L. Dos S., Rocha, A. S Da S., Rodrigues, M.P., Lyra, C De O., \& Ferreira, M, A, F. (2016). Atenção à criança no período neonatal: avaliação do pacto de redução da mortalidade neonatal no Rio Grande do Norte, Brasil. Ciência \&. saúde coletiva, 21 (1), 243252. https://doi.org/10.1590/1413-81232015211.09912014.

Sampaio, Á. R. R., Bousquat, A., \& Barros, C. (2016). Contato pele a pele ao nascer: um desafio para a promoção do aleitamento materno. Epidemiologia e Serviços de Saúde. 25(2), 281-290. https://doi.org/10.5123/S1679-49742016000200007.

Sarli, Y. De O. Di. (2018). Associação entre o tempo de clampeamento do cordão umbilical e icterícia neonatal precoce em recém nascidos a termo. [Dissertação de Mestrado, Universidade Santo Amaro].

Tanus, A. T., \& Carneiro, P. A (2018). O cuidado ao recém-nascido: conhecimento do enfermeiro no âmbito hospitalar. http://repositorio.unis.edu.br/bitstream/prefix/311/1/Amanda.pdf.

Zavaleta, N., \& Robilliard, L.A. (2017). Efecto de la anemia en el desarrollo infantil: consecuencias a largo plazo. Revista Peruana de Medicina Experimental y Salud Publica. 34(4), 716-722. https:// 10.17843/rpmesp.2017.344.3251. 